\title{
Long-Term Forecasting of Internet Backbone Traffic: Observations and Initial Models
}

\author{
Konstantina Papagiannaki, Nina Taft, Zhi-Li Zhang, Christophe Diot
}

\begin{abstract}
We introduce a methodology to predict when and where link additions/upgrades have to take place in an IP backbone network. Using SNMP statistics, collected continuously since 1999, we compute aggregate demand between any two adjacent PoPs and look at its evolution at time scales larger than one hour. We show that IP backbone traffic exhibits visible long term trends, strong periodicities, and variability at multiple time scales.

Our methodology relies on the wavelet multiresolution analysis and linear time series models. Using wavelet multiresolution analysis, we smooth the collected measurements until we identify the overall long-term trend. The fluctuations around the obtained trend are further analyzed at multiple time scales. We show that the largest amount of variability in the original signal is due to its fluctuations at the 12 hour time scale.

We model inter-PoP aggregate demand as a multiple linear regression model, consisting of the two identified components. We show that this model accounts for $98 \%$ of the total energy in the original signal, while explaining $90 \%$ of its variance. Weekly approximations of those components can be accurately modeled with low-order AutoRegressive Integrated Moving Average (ARIMA) models. We show that forecasting the long term trend and the fluctuations of the traffic at the 12 hour time scale yields accurate estimates for at least six months in the future.
\end{abstract}

\section{INTRODUCTION}

IP network capacity planning is a very important task that has received little attention in the research community. The capacity planning theory for traditional telecommunication networks is a well explored area [1], which has limited applicability in a packet-based network such as the Internet. It normally depends on the existence of a traffic matrix, identifying the amount of traffic flowing between any source to any destination of the network under investigation. Moreover, it requires accurate modeling of the incoming traffic, as well as accurate predictions of its future behavior. The above information is then combined in a network simulation to identify the points where future upgrades will be needed.

This approach cannot be used in the environment of an IP backbone network because (i) we do not have a way of measuring or accurately estimating the traffic matrix for such a large scale network, (ii) we do not really know how to model the incoming traffic of a backbone network, and (iii) simulating such a large scale network is typically not feasible.

The current best practice in the area is based on the experience and the intuition of the network operators. Moreover, it usually relies on marketing information regarding

K. Papagiannaki, N. Taft, and C. Diot are with Sprint ATL, 1 Adrian Ct., Burlingame, CA 94010. E-mail: \{dina,nina,cdiot\}@sprintlabs.com. K. Papagiannaki is also with the University College London. Z.-L. Zhang was with Sprint ATL on sabbatical leave from University of Minnesota. E-mail: zhzhang@cs.umn.edu. projected number of customers at different locations within the network. Given provider-specific oversubscription ratios, and traffic assumptions, the operators estimate the effect that the additional customers may have on the network-wide load. The points where link upgrades will take place are selected based on experience, and/or current network state. For instance links that currently carry larger volumes of traffic are likely to be upgraded first.

Our goal is to enhance the above practices using historical network measurements collected with the Simple Network Management Protocol (SNMP). The intuition behind our approach is to use mathematical tools to process historical information and extract trends in the traffic evolution at different time scales. This approach requires the collection of network measurements over long periods of time.

In this paper, we analyze three years of SNMP information collected throughout a major tier-1 IP backbone. Correlating those measurements with topological information, we calculate the traffic aggregate between any two adjacent PoPs and track its evolution over time. We explore the properties of these time series, and propose a methodology that can be applied to forecast network traffic volume months in the future.

Our methodology relies on wavelet multiresolution analysis and linear time series models. Initial observations on the traffic reveal strong periodicities, evident long term trends, and variability at multiple time scales. We use wavelets to smooth out the original signal until we identify the overall long term trend. The fluctuations of the traffic around the obtained trend are further analyzed at multiple time scales. This analysis reveals that $98 \%$ of the energy in the signal is captured by two main components, namely the long term trend, and the fluctuations at the 12 hour time scale. Using the analysis of variance (ANOVA) technique, we further show that a multiple linear regression model containing the two identified components also explains $90 \%$ of the variance.

We model the weekly approximations of the two components using ARIMA models, and develop a prediction scheme that is based on their forecasted behavior. We show that forecasting network backbone traffic based on our model can yield accurate estimates for at least six months in the future. Moreover, with a minimal computational overhead, and by modeling only the long term trend and the fluctuations of the traffic at the 12 hour time scale, we produce estimates which are within $10-15 \%$ of the actual measured behavior.

Our methodology combined with actual backbone traffic measurements leads to different forecasting models for different parts of the network. Our results indicate that different PoP-pairs exhibit different rates of growth and experience 
different types of fluctuations. This illustrates the importance of defining a methodology for deriving models as opposed to developing a single model for inter-PoP aggregate traffic flows.

In section II we present previous efforts at forecasting Internet traffic. Our objectives are presented in section III. In section IV, we present the data analyzed throughout the paper and make some initial observations. Section $\mathrm{V}$ provides an overview of the wavelet multiresolution analysis, along with results of its application on our measurements. Forecasts are derived using linear time series models, presented in section VI. We discuss our findings and future work in section VII. We conclude in Section VIII.

\section{RELATED WORK}

An initial attempt toward long-term forecasting of IP network traffic is described in [2]. The authors compute a single value for the aggregate number of bytes flowing over the NSFNET, and model it using linear time series models. They show that the time series obtained can be accurately modeled with a low-order ARIMA model, offering highly accurate forecasts (within $10 \%$ of the actual behavior) for up to two years in the future.

However, predicting a single value for the future networkwide load is insufficient for capacity planning purposes. One needs to pinpoint the areas in the network where overload may occur in order to identify the locations where future provisioning will be required. Thus per-node or per-link forecasts are required. The authors of [2] briefly address this issue, mentioning that initial attempts toward this direction did not prove fruitful.

Other work in the domain of Internet traffic forecasting typically addresses small time scales, such as seconds or minutes, that are relevant for dynamic resource allocation [3], [4], [5], [6], [7], [8]. To the best of our knowledge, our work is the first to model the evolution of IP backbone traffic at large time scales, and to develop models for long-term forecasting that can be used for capacity planning purposes.

\section{OBJECTIVES}

The "capacity planning" process consists of many tasks, such as addition or upgrade of specific nodes, addition of PoPs, and expansion of already existing PoPs. For the purposes of this work, we use the term "capacity planning" only to refer to the process of upgrading or adding links between two PoPs in the core of an IP network.

The core of an IP network is usually overprovisioned and consists of very high speed links, i.e. OC-48, OC-192. Those links are a rather large part of a network operator's investment and have a provisioning cycle between six and eighteen months. Therefore, the capability to forecast when and where future link additions or upgrades will have to take place would greatly facilitate network provisioning.

In order to address the issue of where upgrades or additions should take place, we measure and forecast aggregate traffic between adjacent PoPs. In that way carriers can determine which pair of PoPs may need additional interconnecting capacity. There are a number of factors that influence when an upgrade is needed. These factors include service level agreements with customers, network policies toward robustness to failures, the rate of failures, etc. We assume that carriers have a method for deciding how many links should interconnect a given pair of PoPs and the acceptable levels of utilization on these links. Once carriers articulate a threshold beyond which traffic levels between PoPs are considered prohibitive, one can schedule an upgrade before these levels are actually exceeded. Our task is to predict when in the future the traffic levels will exceed these acceptable thresholds.

In this work, we use historical information collected continuously since 1999 on the Sprint IP backbone network. There are many factors that contribute to trends and variations in the overall traffic. Our measurements come from a highly dynamic environment reflecting events that may have short or longlived effects on the observed behavior. Some of the events that may have a long-lived effect on the observed behavior include changes in the network topology and in the number of connected customers. These events influence the overall long-term trend, and the bulk of the variability observed. Events that may have a short-lived effect include link failures, breaking news or flash crowd events, as well as denial of service attacks. These events normally have a direct impact on the measured traffic but their effect wears out after some time. As a consequence, they are likely to contribute to the measured time series with values which lie beyond the overall trend. Given that such events are very hard to predict, and are already taken into account in the calculation of the threshold values that will trigger upgrades, as described earlier in this section, we will not attempt to model them in this paper.

\section{Measurements of InTER-PoP AGgRegate Demand}

We now describe the measurements collected and analyzed throughout the paper. We present some initial observations about Internet traffic at time scales larger than one hour. These observations motivate the approach used throughout the rest of the paper.

\section{A. Data collected and analysis}

We collect values for two particular MIB (Management Information Base) objects, incoming and outgoing link utilization in bps, for all the links of all the routers in the Sprint IP backbone throughout a period that spans from 1999 until July 1st 2002. This operation yields traces from more than 2000 links, some of which may not be active anymore. The values collected correspond to an exponentially weighted moving average computed on 10 second link utilization measurements. The exponential weighted average has an average age of 5 minutes and allows for more recent samples to be weighted more heavily than samples earlier in the measurement interval $^{1}$.

Along with the SNMP data, we collect topological information. This information is collected several times per

\footnotetext{
${ }^{1}$ Because these objects belong to a proprietary MIB, we have no further information about how this average value is calculated.
} 
day by an agent downloading configuration information from every router in the network. It contains the names of the routers in each PoP, along with all their active links, and their destinations. Therefore, it allows us to identify those links in the SNMP data set that interconnect specific PoPs in our network.

We correlate the SNMP data with the topological information, and derive aggregate demands, in bps, between any two adjacent PoPs. In this procedure we need to address two issues. Firstly, the collection is not synchronized, i.e. not all links are polled at the same time to avoid overload at the collection station. Secondly, the collection is not reliable (SNMP messages use UDP as their transport protocol), i.e. we may not have one record for each 5 minute interval for every link in the network. As a consequence, the derivation of the aggregate demands is performed as follows:

- For each link in the SNMP data, we identify its source and destination PoP. We use the notation $l_{s d}(k)$ to denote the $k$ th link connecting PoP $s$ to PoP $d$.

- Time is discretized in 90 minute intervals. We denote time intervals with index $t$. The reasons why we selected intervals of 90 minutes are provided in Section V-A.

- The aggregate demand for any PoP-pair $(s, d)$ at time interval $t$ is calculated as the sum of all the records obtained at time interval $t$ from all links $k$ in $\left\{l_{s d}(k)\right\}$, divided by the number of records. This metric gives the average aggregate demand of a link from $\mathrm{PoP} s$ to $\mathrm{PoP}$ $d$ at time interval $t$.

This approach allows us to handle the case of missing values for particular links in the aggregate flow. Moreover, it does not suffer from possible inaccuracies in the SNMP measurements, since such events are smoothed out by the averaging operation. With the aforementioned procedure we obtain 169 time series (one for each pair of adjacent PoPs in our network). For the remainder of the paper we focus our discussion on eight of those. These are the longest traces at our disposal which also correspond to highly utilized paths throughout the network. In the following sections we look into their properties, and devise techniques for forecasting their values in the medium (i.e. months ahead) and long-term future (i.e. 6 months).

\section{B. Initial observations}

In Figure 1 we present the aggregate demand for three PoP pairs in our network. The time series span from October 2000 to July 2002. Those time series correspond to an increasing number of links in time. With vertical bars we denote the time when additional links became active in the aggregate. As can be seen, link additions are rarely preceded by a visible rise in the carried traffic. This behavior is due to the long provisioning cycles.

From the same figure we can see that different PoP pairs exhibit different behaviors as far as their aggregate demand is concerned. A long term trend is clearly visible in the traces. For trace 1, and 5, this trend is increasing with time, while for trace 6 it looks more constant with a sudden shift in January 2002 , that lasts for two months.
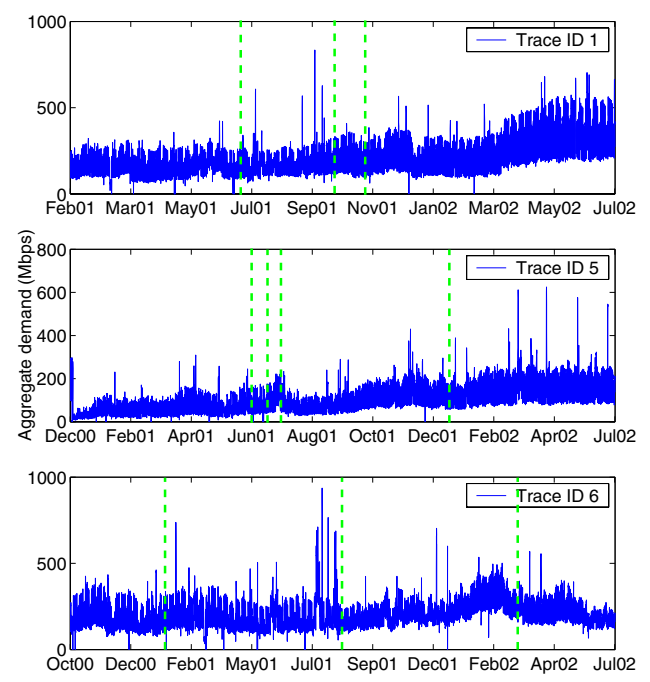

Fig. 1. Aggregate demand for Traces 1, 5, and 6.
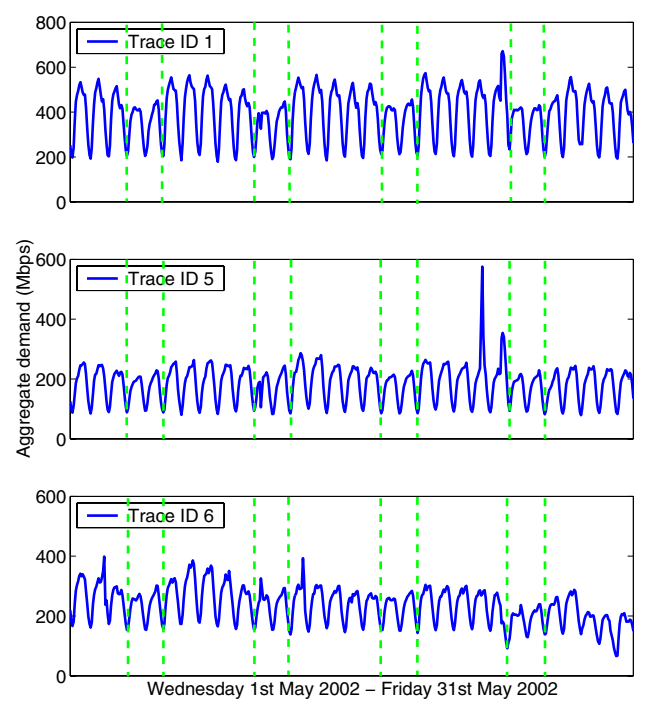

Fig. 2. Aggregate demand in May 2002 for Traces 1, 5, and 6 .

Shorter-term fluctuations around the overall long term trend are also present across all traces, and manifest themselves in different ways. For instance, trace 1 shows an increasing deviation around its long term trend. On the other hand, trace 6 exhibits smaller fluctuations, that look consistent over time.

Regardless of the differences observed in the three traces, one common property is the presence of large spikes throughout them. Notice that those spikes correspond to average values across 90 minutes, which indicate a surge of traffic in that particular interval that is high or constant enough to have a significant effect on a 90 minute average. Those spikes may correspond to link failures, which re-route part of the affected traffic onto this particular path, routing changes, or even denial of service attacks. As mentioned in Section II, we decide to treat those spikes as outliers. This does not mean we ignore the data but simply that we do not attempt to model or predict these spikes.

In Figure 2 we present a detail of Figure 1, which corresponds to the month of May 2002. This figure indicates the 
presence of strong daily and weekly cycles. The drop in traffic during the weekend (denoted by the dashed lines) may be substantial as in trace 1 , smaller as in trace 5, or even nonexistent as in parts of trace 6.
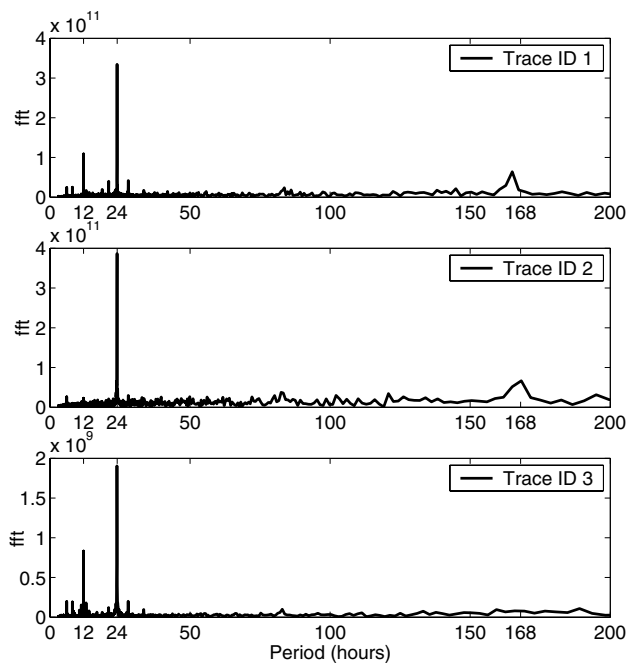

Fig. 3. Fast Fourier Transform for Trace 1, 2, and 3.

From the previous observations it is clear that there are strong periodicities in the data. In order to verify their existence, we calculate the Fourier transform for the eight traces at our disposal. Our results indicate that the most dominant period across all traces is the 24 hour one. Other noticeable periods correspond to 12 , and 168 hours (i.e. weekly period). Figure 3 presents the Fast Fourier Transform for three of our traces. One can notice that when there is a strong 12 hour period, then it is usually stronger than the weekly one. However, depending on the trace, such periods may not even be present ${ }^{2}$.

In summary, initial observations from the collected time series lead to three main findings: 1 ) there is a multi-timescale variability across all traces (traces vary in different ways at different time scales), 2) there are strong periodicities in the data, and 3) the time series exhibit evident long-term trends, i.e. non-stationary behavior. Such findings can be exploited in the forecasting process. For instance, periodicities at the weekly cycle imply that the time series behavior from one week to the next can be predicted. In the next section, we address these three points in our findings.

\section{Multi-Timescale AnAlysis}

In this section, we analyze the collected measurements at different time scales. We show that using the wavelet multiresolution analysis we can isolate the underlying overall trend, and those time scales that significantly contribute to its variability.

\footnotetext{
${ }^{2}$ Trace 5 , and 6 , presented in the previous figures, exhibit similar behavior to Trace 1 . However, for Trace 6 , the weekly period is stronger than the 12 hour one.
}

\section{A. Wavelet MRA overview}

The wavelet multiresolution analysis (MRA) describes the process of synthesizing a discrete signal by beginning with a very low resolution signal (at the coarsest time scale) and successively adding on details to create higher resolution versions of the same signal [9], [10], [11]. Such a process ends with a complete synthesis of the signal at the finest resolution (at the finest time scale). More formally, at each time scale $2^{j}$, the signal is decomposed into an approximate signal (or simply, approximation) and a detailed signal through a series of scaling functions $\phi_{j, k}(t)$ and wavelet functions $\psi_{j, k}(t)$, where $k \in Z$ is a time index at scale $j$. The scaling and wavelet functions are obtained by dilating and translating the mother scaling function $\phi(t), \phi_{j, k}(t)=2^{-j / 2} \phi\left(2^{-j} t-k\right)$, and the mother wavelet function $\psi(t), \psi_{j, k}(t)=2^{-j / 2} \psi\left(2^{-j} t-k\right)$. The approximation is represented by a series of (scaling) coefficients $a_{j, k}$, and the detail by a series of (wavelet) coefficients $d_{j, k}$.

Consider a signal/time series $x(t)$ with $N$ data points at the finest time scale. Using MRA, $x(t)$ can be written as

$$
x(t)=\sum_{k \in Z} a_{p, k} \phi_{p, k}(t)+\sum_{0 \leq j \leq p} \sum_{k \in Z} d_{j, k} \psi_{j, k}(t)
$$

where $p \leq \log N$. The sum with coefficients $a_{p, k}$ represents the approximation at the coarsest time scale $2^{p}$, while the sums with coefficients $d_{j, k}$ represent the details on all the scales between 0 and $p$.

Using the signal processing parlance, the roles of mother scaling and wavelet function $\phi(t)$ and $\psi(t)$ can be described and represented via a low-pass filter $h$ and a high-pass filter $g$ [11]. Consequently, the multiresolution analysis and synthesis of a signal $x(t)$ can be implemented efficiently as a filter bank. The approximation at scale $j,\left\{a_{j, k}\right\}$, is passed through the low-pass filter $h$ and the high-pass filter $g$ to produce the approximation, $\left\{a_{j+1, k}\right\}$, and the detail, $\left\{d_{j+1, k}\right\}$, at scale $j+1$. Note that at each stage, the number of coefficients at scale $j$ is decimated into half at scale $j+1$, due to downsampling. This decimation reduces the number of data points to be processed at coarser time scales, but also leaves some "artifacts" in coarser time scale approximations.

More recently, the so-called à-trous wavelet transform has been proposed, which produces "smoother" approximations by filling the "gap" caused by decimation, using redundant information from the original signal [12], [13]. Under the àtrous wavelet transform, we define the approximations of $x(t)$ at different scales as:

$$
\begin{gathered}
c_{0}(t)=x(t) \\
c_{j}(t)=\sum_{l=-\infty}^{\infty} h(l) c_{j-1}\left(t+2^{j-1} l\right) .
\end{gathered}
$$

where $1 \leq j \leq p$, and $h$ is a low-pass filter with compact support. The detail of $x(t)$ at scale $j$ is given by

$$
d_{j}(t)=c_{j-1}(t)-c_{j}(t) .
$$

Let $d_{j}=\left\{d_{j}(t), 1 \leq t<N\right\}$ denote the wavelet coefficient at scale $j$, and $c_{p}=\left\{c_{p}(t), 1 \leq t<N\right\}$ denote the signal at 
the lowest resolution, often referred to as the residual. Then the set $\left\{d_{1}, d_{2}, \cdots, d_{p}, c_{p}\right\}$ represents the wavelet transform of the signal up to the resolution level $p$, and the signal $x(t)$ can be expressed as an expansion of its wavelet coefficients:

$$
x(t)=c_{p}(t)+\sum_{j=1}^{p} d_{j}(t)
$$

At this point we can justify our decision about averaging our measurements across 90 minutes intervals. We know that using the wavelet MRA we can look into the properties of the signal at time scales $2^{j}$ times coarser than the finest time scale. Furthermore, the collected measurements exhibit strong periodicities at the cycle of 12 and 24 hours. Using 1.5 hours as the finest time scale allows us to look into the behavior of the time series at the periods of interest by observing its behavior at the $3 \mathrm{rd}\left(2^{3} \times 1.5=12\right)$ and 4 th $\left(2^{4} \times 1.5=24\right)$ time scale.

\section{B. MRA application on inter-PoP aggregate demands}

For the smoothing of our data we chose as the low-pass filter $h$ in Equation 3 the $B_{3}$ spline filter, defined by $(1 / 16$, $1 / 4,3 / 8,1 / 4,1 / 16$ ). This is of compact support (necessary for a wavelet transform), and is point-symmetric. Symmetric wavelets have the advantage of avoiding any phase shifts; the wavelet coefficients do not "drift" relative to the original signal. The $B_{3}$ spline filter gives at each resolution level a signal which is much smoother than the one at the previous level without distorting possible periodicities in the data, and preserving the original structure. The $B_{3}$ spline filter has been previously used in time series smoothing in [14], [15], [16].

In order to understand how $c_{j}(t)$ is computed at each time scale $j$, we schematically present in Figure 4 how $c_{1}(5)$, $c_{2}(5)$, and $c_{3}(5)$ are calculated according to Equation 3, and the $B_{3}$ spline filter. Element $c_{1}(5)$ is computed based on the values $c_{0}(t)=x(t)$ at times $(5-2),(5-1), 5$, $(5+1)$, and $(5+2)$. Then, we can calculate $c_{2}(5)$, based on $c_{1}(1), c_{1}(3), c_{1}(5), c_{1}(7)$, and $c_{1}(9)$. Notice that moving toward coarser levels of resolution we need values from the previous resolution level which are farther apart from each other. For this reason, this wavelet transform is called the à-trous wavelet transform, which means "with holes". One important point we should make is that $c_{p}(t)$ is defined for each $t=1,2, \cdots, n$, where $n$ corresponds to 1.5 hour intervals and is limited by the size $N$ of the original signal. According to Equation 3, computing $c_{p}(n)$ requires values of $c_{p-1}$ until time $n+2^{p}$, which iteratively requires values of $c_{p-2}$ until time $n+2^{p-1}$, etc. As a consequence, the calculation of $c_{p}(n)$ requires the original time series $x(t)$ to have $n+\sum_{j=1}^{j=p} 2^{j}$ values. Given that our original signal contains $N$ values, our wavelet coefficients up to the 6th resolution level will contain $n$ values, where $n+\sum_{j=1}^{j=6} 2^{j}=N$, or $n=N-126$.

In Figure 5 and 6 we present the approximation and detail signals for trace 5 at each time scale, when it is analyzed up to resolution level $2^{6}=96$ hours. We chose to use the 6th time scale as our coarsest time scale because it provides a sufficiently smooth approximation signal, capturing the evolution of the time series from one week to the next

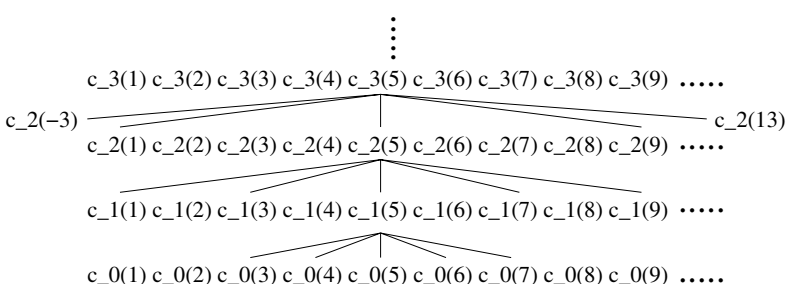

Fig. 4. The à trous wavelet transform.

without the effect of the fluctuations at the 12 and 24 hour time scale. Figure 5 clearly shows how the wavelet MRA smooths out the original signal. Visual inspection of the derived detail signals in Figure 6 further suggests a difference in the amount of variability that each one contributes.

Given the derived decomposition, we calculate the energy apportioned to the overall trend $\left(c_{6}\right)$ and each one of the detail signals. The energy of a signal $y(t), 1 \leq t \leq N$, is defined as $E=\sum_{t=1}^{N} y^{2}(t)$. Table I shows that the overall trend $c_{6}$ accounts for 95 to $97 \%$ of the total energy. Once one subtracts the overall trend from the data, then we notice a substantial difference in the amount of energy distributed among the detail signals. Figure 7 shows that across all eight traces in our study, the maximum amount of energy in the details is always located at the 3rd time scale, which corresponds to the fluctuations across 12 hours. Approximating the original signal as the long term trend, $c_{6}$, and the fluctuations at the 12 hour time scale, $d_{3}$, is further capable of accounting for 97 to $99 \%$ of the total energy (Table I).

\begin{tabular}{ccccc}
\hline Trace ID & 1 & 2 & 3 & 4 \\
\hline$c_{6}$ & $96.07 \%$ & $97.20 \%$ & $95.57 \%$ & $96.56 \%$ \\
\hline$c_{6}+d_{3}$ & $98.10 \%$ & $98.76 \%$ & $97.93 \%$ & $97.91 \%$ \\
\hline \hline Trace ID & 5 & 6 & 7 & 8 \\
\hline$c_{6}$ & $95.12 \%$ & $95.99 \%$ & $95.84 \%$ & $97.30 \%$ \\
\hline$c_{6}+d_{3}$ & $97.54 \%$ & $97.60 \%$ & $97.68 \%$ & $98.45 \%$ \\
\hline \multicolumn{5}{c}{ TABLE I } \\
\end{tabular}

Percentage of total ENERGy IN $c_{6}$, AND $c_{6}+d_{3}$.

In the next section, we look into the properties of the signals derived from the wavelet MRA with respect to the variance they account for in the overall signal.

\section{Analysis of Variance}

As explained in Section V-A, the original signal can be completely reconstructed using the approximation signal at the 6th time scale, and the six detail signals at lower time scales. The model defined in Equation 5 can also be conceived as a multiple linear regression model, where the original signal $x(t)$ is expressed in terms of its coefficients.

The "Analysis of Variance" (ANOVA) technique is a statistical method used to quantify the amount of variability accounted for by each term in a multiple linear regression model [17]. Moreover, it can be used in the reduction process of a multiple linear regression model, identifying those terms 

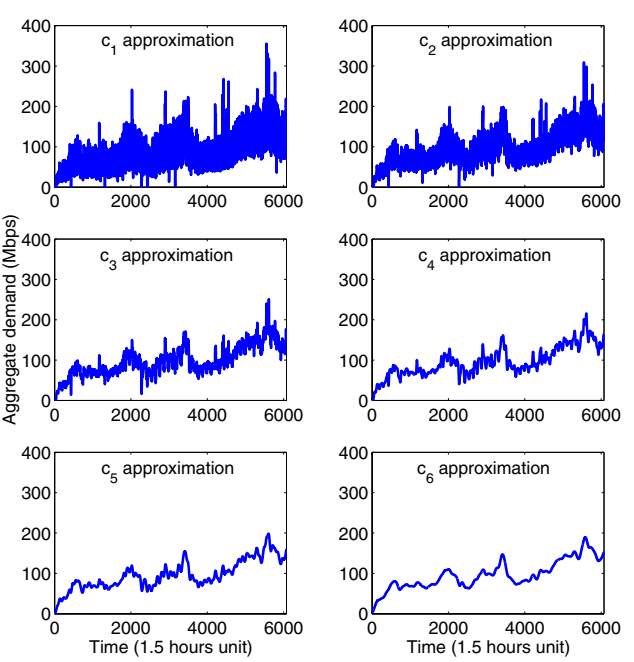

Fig. 5. The approximation signals for trace 5 .
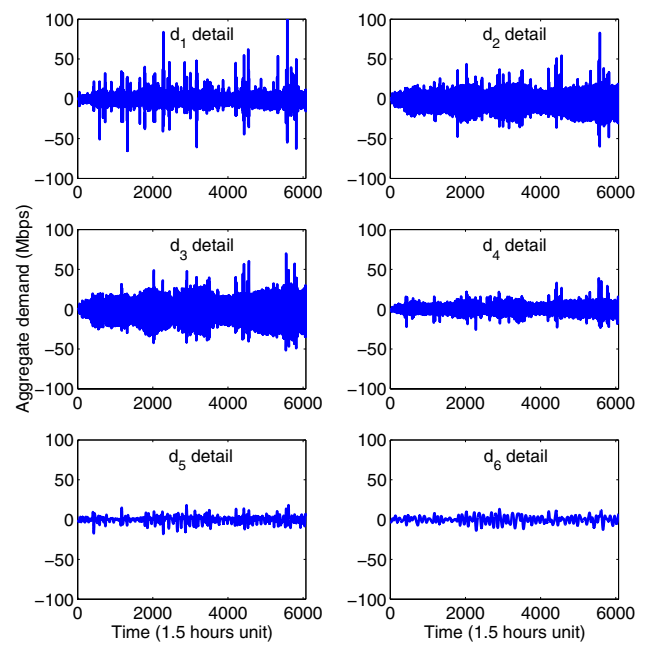

Fig. 6. The detail signals for trace 5 .

in the original model that explain the most significant amount of variance.

Using the ANOVA methodology we calculate the amount of variance in the original signal explained by the 6th approximation signal and each one of the detail signals. The results indicate that the detail signals $d_{1}, d_{2}, d_{5}$, and $d_{6}$ contribute less than $5 \%$ each in the variance of the original signal.

Ideally, we would like to reduce the model of Equation 5, to a simple model of two parameters, one corresponding to the overall long term trend, and a second one accounting for the bulk of the variability. Possible candidates for inclusion in the model, except from the overall trend $c_{6}$, are the signals $d_{3}$ and $d_{4}$. We know that the detail signal $d_{3}$ carries the majority of the energy among all the detail signals. Thus one possible reduced model is the following:

$$
x(t)=c_{6}(t)+\beta d_{3}(t)+e(t)
$$

Using least squares, we calculate the value of $\beta$ for each one of the traces in our disposal. All traces led to a $\beta$ estimate between 2.1 and 2.3 (Table II). Using ANOVA, we test how

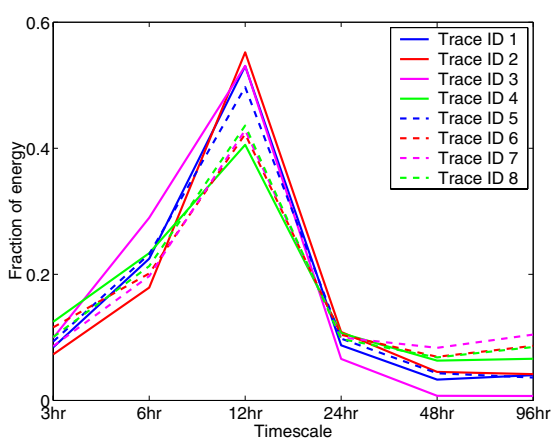

Fig. 7. Energy distribution for the detail signals.

representative the model of Equation 6 is with respect to the proportion of variance it explains [17].

\begin{tabular}{ccccc}
\hline Trace ID & 1 & 2 & 3 & 4 \\
\hline$\beta$ & 2.09 & 2.06 & 2.11 & 2.23 \\
\hline$R^{2}$ & 0.87 & 0.94 & 0.89 & 0.87 \\
\hline \hline Trace ID & 5 & 6 & 7 & 8 \\
\hline$\beta$ & 2.12 & 2.18 & 2.13 & 2.16 \\
\hline$R^{2}$ & 0.92 & 0.80 & 0.86 & 0.91 \\
\hline
\end{tabular}

TABLE II

ANOVA RESULTS FOR ALL EIGHT TRACES.

If $x(t)$ is the observed response, and $e(t)$ is the error incurred in Equation 6, we define $S S X=\sum_{t=1}^{n}(x(t))^{2}$, $S S E=\sum_{t=1}^{n} e(t)^{2}$. The total sum of squares $(S S T)$ is defined as the uncertainty that would be present if one had to predict individual responses without any other information. The best one could do is predict each observation to be equal to the sample mean. Thus we set $S S T=\sum_{t=1}^{n}(x(t)-\bar{x})^{2}$. The ANOVA methodology partitions this variability into two parts. One portion is accounted for by the model. It corresponds to the reduction in uncertainty that occurs when the regression model is used to predict the response. The remaining portion is the uncertainty that remains even after the model is used. We define $S S R$ as the difference between $S S T$ and $S S E$. This difference represents the sum of the squares explained by the regression. The fraction of the variance that is explained by the regression, $S S R / S S T$, determines the goodness of the regression and is called the "Coefficient of Determination", $R^{2}$. The model is considered to be statistically significant if it can account for a large fraction of the variability in the response, i.e. yields large values for $R^{2}$. In Table II, we present the results obtained for the value of $\beta$, and $R^{2}$ for all eight traces.

The reduced model is capable of explaining $80 \%$ to $94 \%$ of the variance in the signal. Moreover, if we decide to include the term $d_{4}$ in the model of Equation 6, the results about $R^{2}$, presented in Table II, are only marginally improved, and increased by 0.01 to 0.04 . 


\section{Summary of findings from MRA and ANOVA}

From the wavelet multiresolution analysis, we draw three main conclusions:

- There is a clear overall long-term trend present in all traces.

- The fluctuations around this long term trend are mostly due to the significant changes in the traffic bandwidth at the time scale of 12 hours.

- The long term trend and the detail signal at the 3rd time scale account for approximately $98 \%$ of the total energy in the original signal.

From the Analysis of Variance, we further conclude that:

- The largest amount of variance in the original signal can be explained by its long term trend $c_{6}$ and the detail signals $d_{3}$, and $d_{4}$ at the time scales of 12 and 24 hours respectively.

- The original signal can be sufficiently approximated by the long term trend and its third detail signal. This model explains approximately $90 \%$ of the variance in the original signal.

Based on those findings, we derive a generic model for our time series, presented in Equation 7. This model is based on Equation 6 , where we set $\beta=3$, for a model valid across the entire backbone.

$$
x^{\prime}(t)=c_{6}(t)+3 d_{3}(t)
$$

\section{E. Implications for modeling}

For forecasting purposes at the time scale of weeks and months, one may not need to accurately model all the short term fluctuations in the traffic. More specifically, for capacity planning purposes, one only needs to know the traffic baseline in the future along with possible fluctuations of the traffic around this particular baseline.

Component $d_{3}(t)$ in the model of Equation 7 is defined for every 90 minutes interval in the measurements capturing in time the short-term fluctuations at the time scale of 12 hours. Given that the specific behavior within a day may not be that important for capacity planning purposes, we calculate the standard deviation of $d_{3}$ within each day. Furthermore, since our goal is not to forecast the exact amount of traffic on a particular day months in the future, we calculate the weekly standard deviation $d t_{3}(j)$ as the average of the seven values computed within each week. Such a metric represents the fluctuations of the traffic around the long term trend from day to day within each particular week.

In Figure 8 we show the aggregate demand for trace 5, as calculated from the SNMP data. In the same figure we plot the long term trend in the data, along with two curves showing the approximation of the signal as the sum of the long term trend plus/minus three times the average daily standard deviation within a week (Equation 7). We see that approximating the original signal in such a way exposes the fluctuations of the time series around its baseline with sufficient accuracy.

Notice that the new signal $d t_{3}$ features one value every week, exposing the average daily standard deviation within

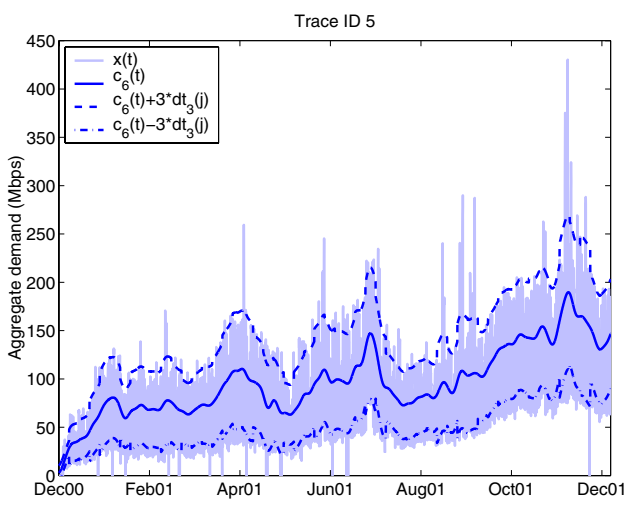

Fig. 8. Approximation of the signal using $c_{6}(t)$ and the average daily standard deviation within a week $d t_{3}(j)$.

the week. Similarly, we can approximate the long term trend with a more compact time series featuring one value for every week. Given that the 6th approximation signal is a very smooth approximation of the original signal, we calculate its average across each week, and create a new time series $l(j)$ capturing the long term trend from one week to the next. The forecasting process will have to predict the behavior of

$$
\hat{x}(j)=l(j)+3 d t_{3}(j),
$$

where $\mathrm{j}$ denotes the index of each week in our trace.

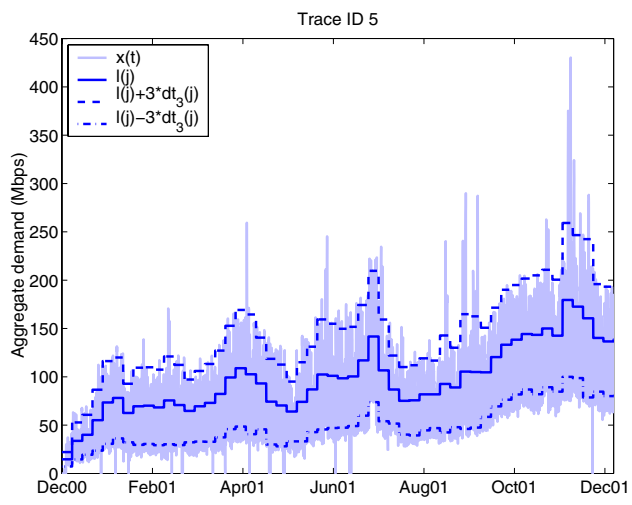

Fig. 9. Approximation of the signal using the average weekly long term trend $l(j)$ and the average daily standard deviation within a week $d t_{3}(j)$.

The resulting signal is presented in Figure 9. We confirm that approximating the original signal using weekly average values for the overall long term trend, and the daily standard deviation results in a model which accurately captures the desired behavior.

In the next section, we introduce the linear time series models, and show how they can help derive forecasts for the two identified components. Once we have those forecasts, we compute the forecast for the original time series and compare it with collected measurements. 


\section{Time Series Analysis Using the ARIMA MOdel.}

\section{A. Overview of linear time series models}

Constructing a time series model implies expressing $X_{t}$ in terms of previous observations $X_{t-j}$, and noise terms $Z_{t}$ which typically correspond to external events. The noise processes $Z_{t}$ are assumed to be uncorrelated with a zero mean and finite variance. Such processes are the simplest processes, and are said to have "no memory", since their value at time $t$ is uncorrelated with all the past values up to time $t-1$.

Most forecasting models described in the literature are linear models. From those models, the most well-known are the "Autoregressive" (AR), "Moving Average" (MA), and "Autoregressive Moving Average" (ARMA) models.

A time series $X_{t}$ is an $\operatorname{ARMA}(\mathrm{p}, \mathrm{q})$ process if $X_{t}$ is stationary and if for every $t$

$X_{t}-\phi_{1} X_{t-1}-\cdots-\phi_{p} X_{t-p}=Z_{t}+\theta_{1} Z_{t-1}+\cdots+\theta_{q} Z_{t-q}$

where $Z_{t} \sim W N\left(0, \sigma^{2}\right)$ and the polynomials $\left(1-\phi_{1} z-\cdots-\right.$ $\left.\phi_{p} z^{p}\right)$ and $\left(1+\theta_{1} z+\cdots+\theta_{q} z^{q}\right)$ have no common factors [18]. If $p=0$, then the model reduces to a pure MA process, while if $q=0$, the process reduces to a pure AR process.

This equation can also be written in a more concise form as:

$$
\phi(B) X_{t}=\theta(B) Z_{t}
$$

where $\phi(\cdot)$, and $\theta(\cdot)$ are the $p^{t h}$ and $q^{t h}$ degree polynomials, and $B$ is the backward shift operator $\left(B^{j} X_{t}=X_{t-j}, B^{j} Z_{t}=\right.$ $\left.Z_{t-j}, j=0, \pm 1, \cdots\right)$.

The ARMA model fitting procedure assumes the data to be stationary. If the time series exhibits variations that violate the stationary assumption, then there are specific approaches that could be used to render the time series stationary. The most common one is what is often called the "differencing operation". We define the lag-1 difference operator $\nabla$ by

$$
\nabla X_{t}=X_{t}-X_{t-1}=(1-B) X_{t},
$$

where $B$ is the backward shift operator as already introduced. If the non stationary part of a time series is a polynomial function of time, then differencing finitely many times can reduce the time series to an ARMA process.

An $\operatorname{ARIMA}(p, d, q)$ model is an $\operatorname{ARMA}(p, q)$ model that has been differenced $d$ times. Thus it has the form:

$$
\phi(B)(1-B)^{d} X_{t}=\theta(B) Z_{t}, Z_{t} \sim W N\left(0, \sigma^{2}\right)
$$

If the time series has a non-zero average value through time, then the previous equation also features a constant term $\mu$ on its right hand side.

\section{B. Time series analysis of the long-term trend and deviation}

In order to model the obtained components $l(j)$ and $d t_{3}(j)$ using linear time series models, we have to separate the collected measurements into two parts: 1) one part used for the estimation of the model parameters, and 2) a second part used for the evaluation of the performance of the selected model. Since our intended application is capacity planning, where traffic demand has to be predicted several months ahead in the future, we select the estimation and evaluation period such that the latter contains six months of data.

For each one of the analyzed traces, we use the measurements collected up to 15th January 2002 for the modeling phase, and the measurements from 16th January 2002 until 1st July 2002 for the evaluation phase. Given that not all time series are of the same duration, the isolation of the last six months for evaluation purposes may lead to specific traces featuring a small number of measurements for the estimation phase. Indeed, after posing this requirement three out of the eight traces in our analysis (Trace 2, 3, and 7) consist of less than six months of information. Such limited amount of information in the estimation period does not allow for model convergence. As a consequence, we continue our analysis on the five traces remaining.

We use the Box-Jenkins methodology to fit linear time series models [18]. Such a procedure involves the following steps: i) determine the number of differencing operations needed to render the time series stationary, ii) determine the values of $p$, and $q$ in Equation 9, iii) estimate the polynomials $\phi$, and $\theta$, and iv) evaluate how well the derived model fits the data. For the model fitting we used both Splus [19] and ITSM [18], and obtained similar results. The estimation of the model parameters is done using Maximum Likelihood Estimation. The best model is chosen as the one that provides the smallest AICC, BIC, and FPE measures [18], while offering the smallest mean square prediction error six months ahead. Due to space constraints, we will not go into details about the metrics used in the quality evaluation of the derived model, and refer the reader to [18]. One point we should emphasize is that metrics like AICC, and BIC not only evaluate the fit between the values predicted by the model and actual measurements, but also penalize models with large number of parameters. Therefore, the comparison of the derived models against such metrics leads to the most parsimonious models fitting the data.

\section{Models for $l(j)$, and $d t_{3}(j)$}

The computed models for the long term trend $l(j)$ indicate that the first difference of those time series (i.e. the time series of their changes) is consistent with a simple MA model with one or two terms (i.e, $d=1, q=1$ or $d=1, q=2$ ), plus a constant value $\mu$ (Table III). The need for one differencing operation at lag 1 , and the existence of term $\mu$ across all the models indicate that the long-term trend across all the traces is a simple exponential smoothing with growth. The trajectory for the long-term forecasts will typically be a sloping line, whose slope is equal to $\mu$. For instance, for trace 1 the longterm forecast will correspond to a weekly increase of 0.5633 Mbps. This forecast corresponds to the average aggregate demand of a link in the aggregate. The weekly increase in the total demand between two adjacent PoPs can thus be estimated through the multiplication of this value with the total number of active links in the aggregate. Given the estimates of $\mu$ across all models in Table III we conclude that all traces exhibit upward trends, but grow at different rates.

Applying the Box-Jenkins methodology on the deviation measurements, we see that for some traces the deviation 


\begin{tabular}{llllcc}
\hline ID & Order & Model & $\mu$ & $\sigma^{2}$ \\
\hline T1 & $(0,1,2)$ & $X(t)=X(t-1)+Z(t)-0.1626 Z(t-1)-0.4737 Z(t-2)$ & $0.5633 \mathrm{E}+06$ & $0.2794 \mathrm{E}+15$ \\
\hline T4 & $(0,1,1)$ & $X(t)=X(t-1)+Z(t)+0.4792 Z(t-1)$ & $0.4155 \mathrm{E}+06$ & $0.1339 \mathrm{E}+15$ \\
\hline T5 & $(0,1,1)$ & $X(t)=X(t-1)+Z(t)+0.1776 Z(t-1)$ & $0.2301 \mathrm{E}+07$ & $0.1516 \mathrm{E}+15$ \\
\hline T6 & $(0,1,2)$ & $X(t)=X(t-1)+Z(t)-0.3459 Z(t-1)-0.4578 Z(t-2)$ & $0.7680 \mathrm{E}+06$ & $0.6098 \mathrm{E}+15$ \\
\hline T8 & $(0,1,1)$ & $X(t)=X(t-1)+Z(t)+0.2834 Z(t-1)$ & $0.2021 \mathrm{E}+07$ & $0.1404 \mathrm{E}+16$ \\
\hline
\end{tabular}

TABLE III

ARIMA MODELS FOR THE LONG TERM TREND.

\begin{tabular}{llllll}
\hline ID & Order & Model & $\mu$ & $\sigma^{2}$ \\
\hline T1 & $(0,1,1)$ & $X(t)=X(t-1)+Z(t)-0.6535 Z(t-1)$ & $0.3782 \mathrm{E}+05$ & $0.2024 \mathrm{E}+14$ \\
\hline T4 & $(2,0,0)$ & $X(t)=0.8041 X(t-1)-0.3055 X(t-2)+Z(t)$ & $0.1287 \mathrm{E}+08$ & $0.7295 \mathrm{E}+13$ \\
\hline T5 & $(0,1,1)$ & $X(t)=X(t-1)+Z(t)-0.1493 Z(t-1)$ & $0.3094 \mathrm{E}+06$ & $0.8919 \mathrm{E}+13$ \\
\hline T6 & $(3,0,0)$ & $X(t)=0.3765 X(t-1)-0.1964 X(t-2)-0.2953 X(t-3)+Z(t)$ & $0.2575 \mathrm{E}+08$ & $0.3057 \mathrm{E}+14$ \\
\hline T8 & $(0,1,1)$ & $X(t)=X(t-1)+Z(t)-0.5565 Z(t-1)$ & $0.3924 \mathrm{E}+05$ & $0.4423 \mathrm{E}+14$ \\
\hline
\end{tabular}

TABLE IV

ARIMA MODELS FOR THE WEEKLY DEVIATION.

$d t_{3}(j)$ can be expressed with simple AR models (Trace 4, and 6), while the remaining can be accurately modeled as MA processes after one differencing operation (Table IV). Therefore, the deviation for traces 1,5 , and 8 increases with time (at rates one order of magnitude smaller than the increase in their long term trends), while the deviation for traces 4 , and 6 can be approximated with a weighted moving average, which indicates slower evolution. These results confirm earlier observations on Figure 1 in Section IV-B.

From the previous tables we see that one cannot come up with a single network-wide forecasting model for the interPoP aggregate demand. Different parts of the network grow at different rates (long-term trend), and experience different types of variation (deviation from the long-term trend). Our methodology extracts those trends from historical measurements and can identify these PoP pairs in the network that exhibit higher growth rates and thus may require additional capacity in the future.

At this point we should note that the Box-Jenkins methodology could also have been applied on the original time series $x(t)$. However, given the existence of three strong periods in the data (which would require a seasonal ARIMA model with three seasons [18]), the variability of the time series at multiple time scales, the existence of outliers, and the size of the original time series, such an approach leads to highly inaccurate forecasts, while being extremely computationally intensive. Our technique is capable of isolating the overall long term trend and identifying those components that significantly contribute to its variability. Predictions based on weekly approximations of those components provide accurate estimates with a minimal computational overhead. All our forecasts were obtained in seconds.

In the next section, we use the derived models for the weekly prediction of the aggregate traffic demands. Our forecasts are compared against actual measurements.

\section{Evaluation of forecasts}

Using our models we predict a baseline aggregate demand for a particular week in the future, along with possible deviations around it. The overall forecast for the inter-PoP aggregate demand is then calculated based on Equation 8. We constrain ourselves to the upper limit alone, since this is the value that would be used for capacity planning purposes.

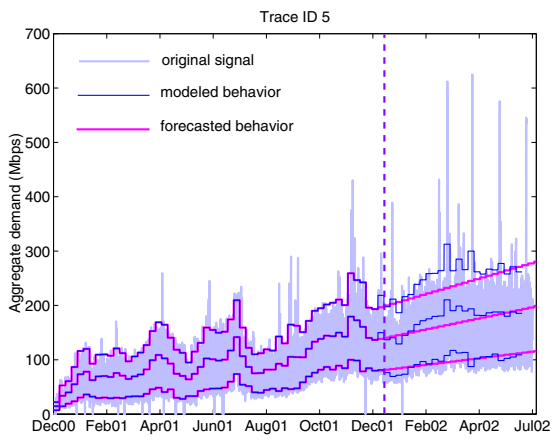

Fig. 10. Six month forecast for Trace 5 .

In Figure 10, we present the time series collected until July 1st 2002. On the same figure we present the modeled behavior in the estimation period, and the forecasts in the evaluation period $^{3}$. From visual inspection of the presented plot, one can conclude that the proposed methodology behaves very well for this particular trace.

In order to be able to quantify the quality of the predictions with respect to the observed behavior, we proceed as follows:

- We apply the MRA on the measurements in the evaluation period.

- We calculate the long term trend $l(j)$ and weekly deviation $d t_{3}(j)$ for each week in the same period.

- We compute $\hat{x}(j)$ based on Equation 8 .

\footnotetext{
${ }^{3}$ In Figures 10, 12 and 13, the vertical dashed line indicates the beginning of the forecasting period.
} 
- Lastly, we calculate the error in the derived forecast as the forecasted value minus $\hat{x}(j)$, divided by $\hat{x}(j)$.

In Figure 11 we present the relative error between the derived forecast and $\hat{x}(j)$ for each week in the evaluation period. Negative error implies that the actual demand was higher than the one forecasted. As can be seen from the figure, the forecasting error fluctuates with time, but is centered around zero. This means that on average we neither underestimate nor overestimate the aggregate demand. More specifically, we see that 24 weeks in the future our prediction error is $4 \%$. The average prediction error across weeks is $-3.6 \%$. Lastly, across all five traces, the average absolute relative prediction error is lower than $15 \%$.

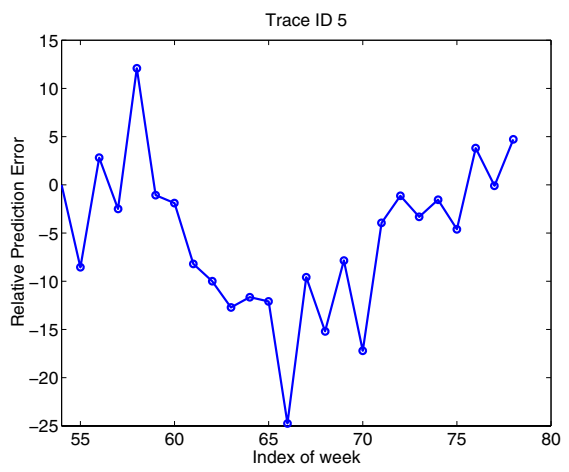

Fig. 11. Weekly relative prediction error for Trace 5.

Notice that our forecasting models can be used to predict demand for more than six months in the future, and identify when the forecasted demand will exceed the operational thresholds that will trigger link upgrades (as explained in section III). In that case though forecasts should be used with caution. As is the case with any forecasting methodology, the farther ahead in the future one attempts to predict, the larger the error margin that should be allowed.

\section{DISCUSSION AND FUTURE WORK}

In the previous sections, we modeled the average aggregate demand between any two adjacent PoPs based on its long term trend and possible short-term fluctuations around it. The long term trend in the data is sensitive to long-lasting routing changes, topological changes, and market conditions that dictate the behavior of the users in the Internet.

Consequently, there will be cases when the fitted ARIMA models will have to be re-estimated in order to adjust to changes in the environment. One example is trace 1. This particular trace is the worst-performing trace in our dataset exhibiting a dramatic change in its overall long term trend right after April 2002. Our analysis uses data until January 15, 2002 to estimate the parameters of the ARIMA models, missing the change in trend that occurs in April. As a result our forecasts are highly accurate until April 2002 (with an average relative prediction error of $1 \%$ ) but substantially smaller than the ones observed between April and July 2002 (Figure 12).

Nonetheless, if re-estimation of the model parameters is triggered at the end of April 2002, then the forecasts are

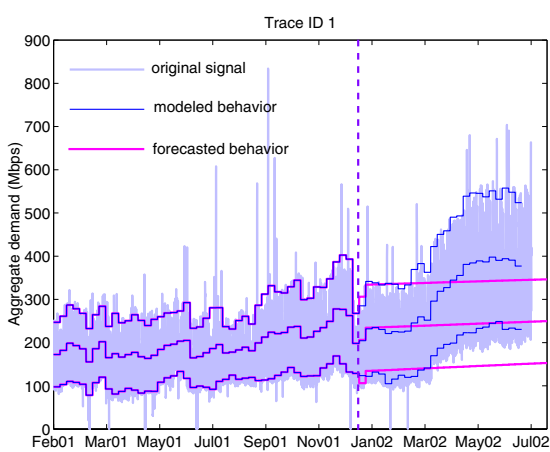

Fig. 12. Weekly prediction for Trace 1.

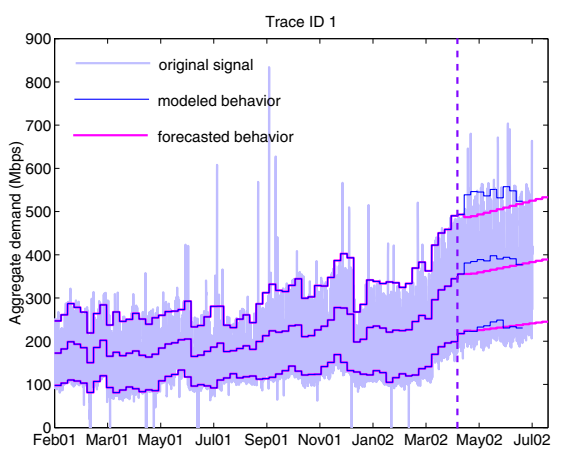

Fig. 13. Adjusted weekly prediction for Trace 1 .

much closer to the measured behavior, and within 7\% (Figure 13). Due to lack of SNMP information beyond July 2002, we cannot evaluate the accuracy of the new predictions in the six months horizon.

Future work will address the issue of detecting changes in the overall trend. Once such a change is detected, then our models can be applied as already shown. In fact, such a detection should not necessarily be automated. Network operators themselves could trigger the re-estimation of the derived ARIMA models a few months after significant topological and/or routing configuration changes have taken place. Within the topological changes, we also include the activation of new high-capacity customer links which may have a significant effect on the load in the core. Such changes would usually be known ahead of time. Consequently, in future work we also intend to incorporate marketing information in our model and evaluate its effect on our forecasts.

\section{CONCLUSIONS}

We presented a methodology for predicting when and where link upgrades/additions have to take place in the core of an IP network. We measured aggregate demand between any two neighboring PoPs in the core of a major tier-1 IP network, and analyzed its evolution at time scales larger than one hour.

We showed that the derived time series exhibit strong periodicities at the cycle of 12 , and 24 hours, as well as one week. Moreover, they experience variability at multiple time scales, and feature distinct overall long-term trends.

Using wavelet MRA, we isolated the overall long term trend, and analyzed variability at multiple time scales. We 
showed that the largest amount of variability in the signal comes from its fluctuations at the 12 hour time scale. Our analysis indicates that a parsimonious model consisting of those two identified components is capable of capturing $98 \%$ of the total energy in the original signal, while explaining $90 \%$ of its variance. The resulting model is capable of revealing the behavior of the network traffic through time, filtering shortlived events that may cause traffic perturbations beyond the overall trend.

We showed that the weekly approximations of the two components in our model can be accurately modeled with loworder ARIMA processes. Our results indicate that different parts in the network grow at different rates, and may also experience increasing deviations from their overall trend, as time progresses. We further showed that calculating future demand based on the forecasted values for the two components in our traffic model yields highly accurate estimates. Our average relative forecasting error is less than $15 \%$ for at least six months in the future.

Due to the properties of the collected time series direct application of traditional time series analysis techniques proves cumbersome, computationally intensive and prone to error. Our methodology is simple to implement, and can be fully automated. Moreover, it provides accurate forecasts for at least six months in the future with a minimal computational overhead. In this paper, we demonstrated its performance within the context of capacity planning. However, multiresolution analysis of the original signal and modeling of selected approximation and detail signals using ARIMA models could possibly provide accurate forecasts for the behavior of the traffic at other time scales, such as from one day to the next or at a particular hour on a given day in the future. These forecasts could be useful for other network engineering tasks, like scheduling of maintenance windows or large database network backups.

\section{ACKNOWLEDGMENTS}

We would like to thank Rene Cruz, Jean-Chrysostome Bolot, Patrick Thiran, Jean-Yves Le Boudec, Chen-Nee Chuah, Jon Crowcroft, and Saleem Bhatti for their valuable feedback.

\section{REFERENCES}

[1] H. Leijon, "Basic Forecasting Theories: A Brief Introduction," ITU, Tech. Rep., Nov. 1998.

[2] N. K. Groschwitz and G. C. Polyzos, "A Time Series Model of Long-Term NSFNET Backbone Traffic," in IEEE ICC'94, 1994. [Online]. Available: http://www-cse.ucsd.edu/ polyzos/ICC.94.ps

[3] S. Basu and A. Mukherjee, "Time Series Models for Internet Traffic," in 24th Conf. on Local Computer Networks, Oct. 1999, pp. 164-171.

[4] J. Bolot and P. Hoschka, "Performance Engineering of the World Wide Web: Application to Dimensioning and Cache Design," in 5th International World Wide Web Conference, May 1996.

[5] K. Chandra, C. You, G. Olowoyeye, and C. Thompson, "Non-Linear Time-Series Models of Ethernet Traffic," CACT, Tech. Rep., June 1998.

[6] R. A. Golding, "End-to-end performance prediction for the Internet," CISB, University of California, Santa Cruz, Tech. Rep. UCSC-CRL92-96, June 1992. [Online]. Available: ftp://ftp.cse.ucsc.edu/pub/tr/ucsccrl-92-26.ps.Z

[7] A. Sang and S. Li, "A Predictability Analysis of Network Traffic," in INFOCOM, Tel Aviv, Israel, Mar. 2000. [Online]. Available: http://www.ieee-infocom.org/2000/papers/47.ps
[8] R. Wolski, "Dynamically Forecasting Network Performance Using the Network Weather Service," in Journal of Cluster Computing, 1999.

[9] I. Daubechies, "Ten Lectures on Wavelets," in Cbms-Nsf Regional Conference Series in Applied Mathematics, vol. 61, 1992.

[10] S. Mallat, "A theory for multiresolution signal decomposition: the wavelet representation," in IEEE Transactions on Pattern Analysis and Machine Intelligence, vol. 11, no. 7, July 1989, pp. 674-693.

[11] J. Walker, A primer on wavelets and their scientific applications. Chapman \& Hall, 1999.

[12] G. Nason and B. Silverman, "The Stationary Wavelet Transform and some Statistical Applications," in Lecture Notes in Statistics: Wavelets and Statistics, 1995, pp. 281-300. [Online]. Available: citeseer.nj.nec.com/nason95stationary.html

[13] M. Shensa, "The Discrete Wavelet Transform: Wedding the À Trous and Mallat Algorithms," in IEEE Transactions on Signal Processing, vol. 40, no. 10,1992 , pp. 2464-2482.

[14] J.-L. Starck and F. Murtagh, "Image restoration with noise suppression using the wavelet transform," Astronomy and Astrophysics, vol. 288, pp. 342-348, 1994

[15] A. Aussem and F. Murtagh, "Web traffic demand forecasting using wavelet-based multiscale decomposition," in International Journal of Intelligent Systems, vol. 16, 2001, pp. 215-236.

[16] P. Yu, A. Goldberg, and Z. Bi, "Time Series Forecasting using Wavelets with Predictor-Corrector Boundary Treatment," in 7th ACM SIGKDD International Conference on Knowledge Discovery and Data Mining, San Francisco, CA, 2001.

[17] R. Jain, The art of computer systems performance analysis: techniques for experimental design, measurement, simulation, and modeling. New York: John Wiley, 1991.

[18] P. Brockwell and R. Davis, Introduction to Time Series and Forecasting. Springer, 1996.

[19] W. N. Venables and B. D. Ripley, Modern Applied Statistics with SPLUS. Springer, 1999. 